\title{
Computed Tomographic Sarcopenia in Pancreatic Cancer: Further Utilization to Plan Patient Management
}

\author{
Mustafa Jalal $^{1}$ (D) . Jennifer A. Campbell ${ }^{1}$ (D) . Jonathan Wadsley ${ }^{2}$ (D) Andrew D. Hopper ${ }^{1}$ (D)
}

Accepted: 13 July 2021 / Published online: 22 July 2021

(c) The Author(s) 2021

\begin{abstract}
Purpose The presence of a sarcopenia adversely affects the prognosis of patients with pancreatic cancer. There is an emerging role for using computed tomography (CT) to calculate skeletal muscle index (SMI) and the presence of sarcopenia. The aim of this study was to assess if detecting 'computed tomographic sarcopenia' is feasible and can contribute to the management of patients with locally advanced pancreatic cancer (LAPC).

Methods Patients diagnosed with LAPC referred for endoscopic ultrasound-guided biopsy (EUS-B) by our regional cancer network were identified. Age, body mass index (BMI), and Eastern Cooperative Oncology Group performance status (ECOG-PS) were noted. CT images were analysed for SMI and the presence of sarcopenia. Decision outcomes on receiving chemotherapy or not were collected from the regional oncology database.

Results In total, 51/204 (25\%) patients with LAPC who underwent EUS-B were not given chemotherapy and received best supportive care (BSC) only. The prevalence of sarcopenia $(p=0.0003)$, age $\geq 75$ years old $(p=0.03)$, and ECOG-PS $2-3(p=0.01)$ were significantly higher in the patients receiving BSC only. Logistic regression analysis demonstrated that SMI was the only independent associated factor identifying patients with LAPC who were treated with BSC only and not chemotherapy after adjusting for age and ECOG-PS.

Conclusion Our study has shown that computed tomographic skeletal muscle analysis at the time of a diagnostic CT for patients with pancreatic cancer is feasible and can detect sarcopenia and malnourished patients who are much less likely to take up chemotherapy. These patients could be triaged to oncology assessment prior to EUS-B to avoid unnecessary investigations.
\end{abstract}

Keywords Pancreatic cancer · Skeletal muscle $\cdot$ Sarcopenia $\cdot$ Palliative chemotherapy $\cdot$ Best supportive care

\section{Introduction}

Pancreatic adenocarcinoma (PDAC) is the 11th most common cancer in the UK, and it accounts for $3 \%$ of cancer cases per year [1]. PDAC has a poor prognosis with $80 \%$ of patients presenting with local or advanced disease at diagnosis [2]. Recent reports have demonstrated that the loss of skeletal muscle mass during neo-adjuvant chemotherapy for borderline resectable pancreatic cancer was associated with poor survival and lower resection rates [3, 4]. A low

Mustafa Jalal

mustafa.jalal@nhs.net

$1 \quad$ Academic Department of Gastroenterology, Royal Hallamshire Hospital, Glossop Road, Sheffield S10 2JF, UK

2 Department of Oncology, Weston Park Hospital, Whitham Road, Sheffield S10 2SJ, UK skeletal muscle mass and strength are defined as sarcopenia [5]. Sarcopenia has a reported prevalence of 25-63\% in PDAC, and it has been shown to impact adversely on the prognosis of patients undergoing surgical resection or palliative therapy [6-11]. Sarcopenia can still occur in patient with a raised body mass index (BMI), and the combination of both is given the term 'sarcopenic obesity' which was shown to adversely impact on survival in PDAC [6].

Given its aggressive course, pancreatic cancer has been described as a 'medical emergency', and patients undergo a rapid sequence of tests to stage and confirm the diagnosis once made [12]. Unfortunately, only $8 \%$ of patients present with resectable pancreatic cancer; therefore, the remaining are considered for palliative chemotherapy only [1, 13]. For metastatic or locally advanced pancreatic cancer (LAPC) disease, endoscopic ultrasound-guided biopsy (EUS-B) is recommended prior to palliative chemotherapy [14-16]. 
However, the availability of EUS-B services is often limited to tertiary centres and university hospitals requiring some patients to travel significant distances for an invasive procedure that requires significant sedation [17]. It has been recorded by cancer support charities that, in all patients with local and metastatic advanced cancer who undergo a biopsy from the primary or a metastatic site, only $28 \%$ of patients go on to receive chemotherapy [18]. Therefore, an EUS-B can be an unnecessary and onerous procedure for many patients with PDAC and delay them receiving supportive and beneficial treatments that focus on nutrition, pain, and psychological support termed best supportive care (BSC) $[13,19,20]$.

There is an emerging role for combining computed tomography $(\mathrm{CT})$ scan with image analysis software to measure body composition and assess presence of sarcopenia [21]. Patients undergo CT scanning as part of the standard care for suspected PDAC [22] and, therefore, presents the opportunity to gain prognostic information about a patient. The aim of this study was to assess if presence of sarcopenia on the diagnostic CT scan could predict patients who do not go on to receive palliative chemotherapy in LAPC after a EUS-B.

\section{Methods}

Prospective identification of patients with diagnosis of LAPC referred for EUS-B by the regional cancer network between Jan 2016 and Dec 2018. Patient information collected included the following: sex, age, BMI, and Eastern Cooperative Oncology Group performance status (ECOGPS). Decision outcomes on receiving chemotherapy or not were collected from the regional oncology database.

\section{Computerised Tomography Skeletal Muscle Mass Measurements}

Diagnostic CT images (Canon Aquilion One Scanners, $120 \mathrm{kV}$, slice thickness $1 \mathrm{~mm}$ ) using intravenous contrast and examined in the venous phase were analysed for sarcopenia. Skeletal muscle was analysed from a single axial CT image at the level of the third lumbar vertebra using a commercially available software (sliceOmatic V5, Tomovision, Quebec, Canada). Images were anonymised, given a study number, and downloaded in Digital Imaging and Communications in Medicine (DICOM) format.

Using sliceOmatic, skeletal muscle area was identified using Hounsfield unit (HU) thresholds $(-29$ to +150$)$ [8]. Cross-sectional area $\left(\mathrm{cm}^{2}\right)$ of skeletal muscle (psoas, erector spinae, quadratus lumborum, transversus abdominis, external and internal oblique abdominis, and rectus abdominis muscles) was measured and identified as shown in Fig. 1. The surface area of skeletal muscle was normalised using the square of patient's height to calculate skeletal muscle index (SMI) expressed as $\left(\mathrm{cm}^{2} / \mathrm{m}^{2}\right)$. Sarcopenia was defined as a SMI $<41 \mathrm{~cm}^{2} / \mathrm{m}^{2}$ for women, and SMI $<43 \mathrm{~cm}^{2} / \mathrm{m}^{2}$ if BMI $<25 \mathrm{~kg} / \mathrm{m}^{2}$ or $<53 \mathrm{~cm}^{2} / \mathrm{m}^{2}$ if BMI $\geq 25 \mathrm{~kg} / \mathrm{m}^{2}$ for men [23].

Using previously accepted definitions, sarcopenic obesity in this study was referred to the presence of both sarcopenia and a BMI $\geq 25 \mathrm{~kg} / \mathrm{m}^{2}$ [24].

\section{Statistical Analysis}

Fischer's exact test and Mann Whitney $U$ test were used to compare categorical and continuous variables. Binary logistic regression was used to assess association of variables with non-uptake of chemotherapy. A $p$ value $<0.05$ was considered statistically significant. Statistical analyses were performed using SPSS (IBM SPSS statistics version 25, Chicago, Illinois, USA). The study was approved by local research ethics committee (IRAS 301,193; STH 21,885).

\section{Results}

A total of 204 patients with LAPC referred for EUS-B were included, and 114 males with median age 69 (42-84) years. The site of the cancers identified was recorded in the head $66.2 \%$ (135), body $30.4 \%$ (62), and tail 3.4\% (7) of the pancreas.

Sarcopenia was present in 54.4\% (111) patients and was significantly higher in female $63.3 \%$ compared to male patients $47.4 \%, p=0.03$. Sarcopenic obesity was present in $27 \%$ (55). The majority of patients had PS $\leq 185.8 \%$ (175) compared to PS $\geq 214.2 \%$ (29).

Of the 204 patients recruited, $75 \%$ (153) received at least one dose of a chemotherapy agent compared to $25 \%$ (51) who had BSC (Table 1). SMI was significantly lower in the BSC group compared to chemotherapy group. The presence of sarcopenia, age $\geq 75$ years old, and ECOGPS 2-3 were more common in BSC. There were no significant differences in comparing the site of cancer, BMI, BMI $<20 \mathrm{~kg} / \mathrm{m}^{2}$, or sarcopenic obesity between the BSC and chemotherapy groups.

Performing logistic regression analysis demonstrated that SMI was the only associated factor identifying patients to choose BSC $(p=0.04)$ and not uptake chemotherapy after adjusting for age, and ECOG-PS. The estimated odds ratio favoured a decrease of nearly $4.5 \%$ for opting into BSC for every 1-unit increase of SMI. 

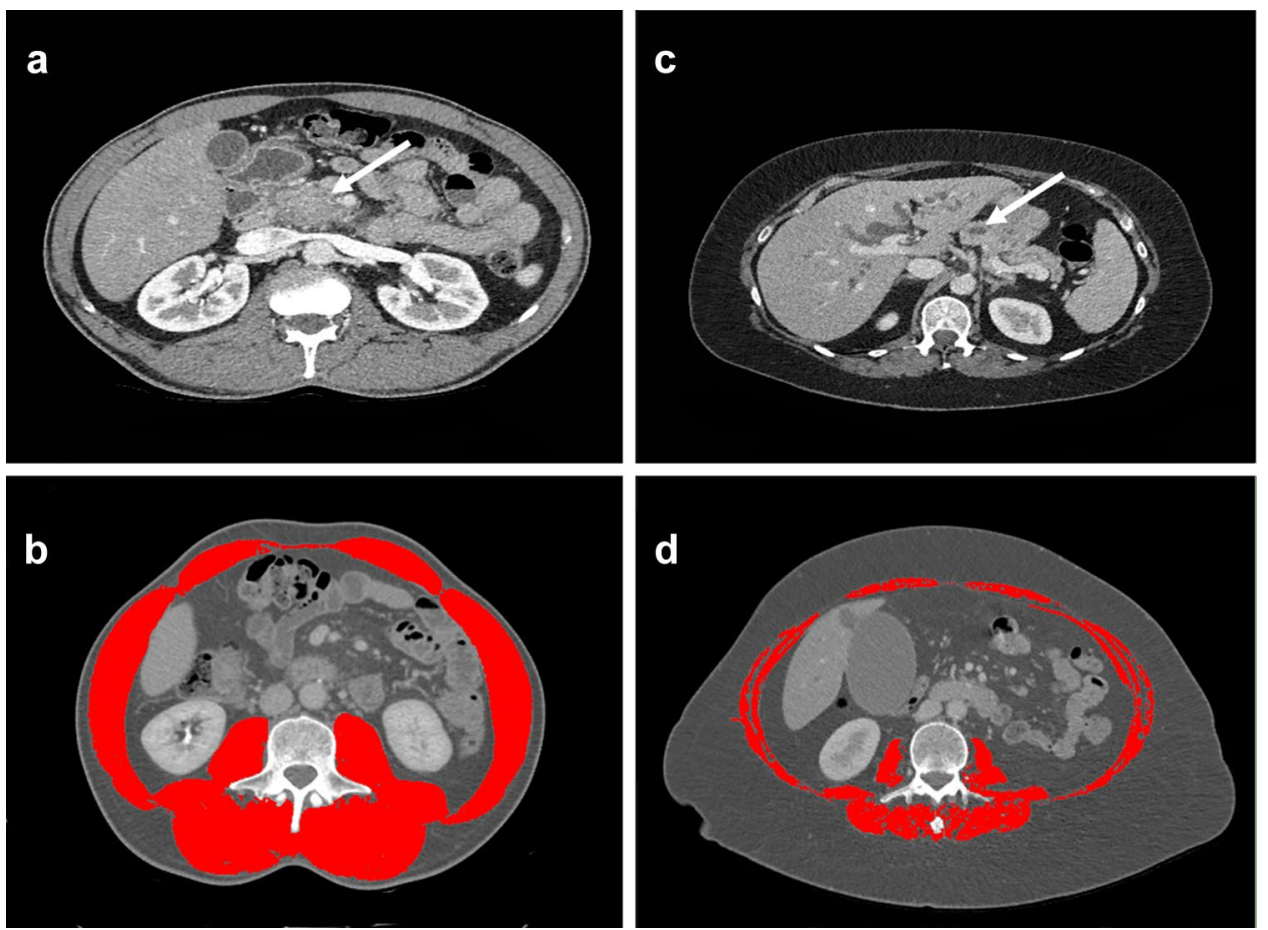

Fig. 1 Computed tomographic skeletal muscle analysis in patients with locally advanced pancreatic cancer. Patient 1 : a locally advanced tumour visible in the uncinate process (white arrow), despite patient's relatively low BMI $\left(21.6 \mathrm{~kg} / \mathrm{m}^{2}\right)$ computed tomographic muscle mass analysis at the L3 level, (b) showed a normal ratio of skeletal muscle (highlighted red) SMI $50.1 \mathrm{~cm}^{2} / \mathrm{m}^{2}$ with the patient receiving chemotherapy. Patient 2: c with a locally advanced pan-

\section{Discussion}

We have shown that SMI measurement at the time of a diagnostic CT for pancreatic cancer is feasible and can creatic cancer in the head and a dilated main pancreatic duct (white arrow), despite the high BMI $32.1 \mathrm{~kg} / \mathrm{m}^{2}$ computed tomographic muscle mass analysis demonstrated a low SMI $31.3 \mathrm{~cm}^{2} / \mathrm{m}^{2}$ consistent with sarcopenia, the patient did not go on to receive chemotherapy. The presence of a high BMI $>25 \mathrm{~kg} / \mathrm{m}^{2}$ and sarcopenia is termed 'sarcopenic obesity' [24]

identify sarcopenia. We have also shown that the presence of 'computed tomographic sarcopenia' objectively identifies malnourished patients who are much less likely to take up chemotherapy. To our knowledge, the association

Table 1 Comparison of the demographics and anthropometric assessments for patients with locally advanced pancreatic cancer receiving best supportive care only or chemotherapy

\begin{tabular}{llll}
\hline Parameter & $\begin{array}{l}\text { Best supportive care } \\
\%(n) \text { or median (range) }\end{array}$ & $\begin{array}{l}\text { Chemotherapy } \\
\%(n) \text { or median (range) }\end{array}$ \\
\hline Number of patients & $25 \%(51)$ & $75 \%(153)$ & $P$ Value \\
Age, years & $71(49-84)$ & $68(42-83)$ & $19.6 \%(30)$ \\
Age $\geq 75$ years & $37.3 \%(18)$ & $59.5 \%(91)$ & $\mathbf{0 . 0 2}$ \\
Male & $45.1 \%(23)$ & $40.5 \%(62)$ & NS \\
Female & $54.9 \%(28)$ & $89.5 \%(137)$ & $10.5 \%(16)$ \\
ECOG-PS 0-1 & $74.5 \%(38)$ & $25(15.4-50.8)$ \\
ECOG-PS 2-3 & $25.5 \%(13)$ & $8.5 \%(13)$ & $\mathbf{0 . 0 1}$ \\
BMI kg/m & $23.6(17.7-42.2)$ & $47.8 \%(73)$ & $\mathrm{NS}$ \\
BMI $<20 \mathrm{~kg} / \mathrm{m}^{2}$ & $15.7 \%(8)$ & $44.2(26.5-80.9)$ \\
Sarcopenia & $76.5 \%(38)$ & $27.5 \%(42)$ \\
SMI & $40.5(26.3-60)$ & $\mathrm{NS}$ \\
Sarcopenic obesity & $25.5 \%(13)$ & $\mathbf{0 . 0 0 0 3}$ \\
\hline
\end{tabular}

NS not significant, ECOG-PS Eastern Cooperative Oncology Group performance status, BMI body mass index, SMI skeletal muscle index 
between sarcopenia and low uptake of palliative chemotherapy in patients with LAPC has not been described before and could facilitate a patient's care pathway. The average time for SMI measurement for a single patient was 2.5 min so could make up part of an initial clinical or multidisciplinary team assessment.

A notable benefit of SMI assessment for patients with PDAC from the diagnostic CT is that it performs an early, objective nutritional assessment tool. Patients presenting with suspected malnutrition can be assessed with alternative methods such as percentage weight loss [25], but a single BMI measurement is of limited diagnostic use on its own [26]. Malnutrition screening tools are available which do have a high inter-rater reliability $(\kappa=0.67-1.00)$ [27-29], and recording specific anthropometric measurements is possible with serial readings [30, 31]. However, alternative nutritional assessment methods should also be interpreted with caution in an obese population [31, 32]: our study showed that nearly half of our patients were overweight, and approximately 1 in 4 patients had sarcopenic obesity. The average weight-losing patient in PDAC is overweight which highlights the reported link with obesity $[6,33]$. A further advantage of sarcopenia assessment is that patients with sarcopenic obesity may be at higher risk of chemotherapy toxicity due to dosing currently being based on body surface area which does not take into account body composition [22]. The benefits of nutritional assessment and subsequent intervention have been demonstrated in one study showing an independent association with survival among patients with unresectable pancreatic cancer (hazard ratio 2.12) regardless of chemotherapy treatment [19].

Despite tissue diagnosis, 51 (25\%) of our patients with LAPC did not receive chemotherapy despite undergoing a procedure to obtain a biopsy specifically to be considered for this purpose. Although still high, this proportion is much lower than other reported data would predict [1], and lower than our initial data collection published in abstract form [34]. Our findings would be supported by our exclusion of patients with metastatic disease (who would undergo liver biopsy locally) and our cancer network awareness of the initial abstract findings prompting attempts to improve patient assessment prior to EUS-B referral.

The subjective assessment of patients ECOG-PS is challenging and can alter quickly; in this study, it proved to be a limitation, as we did not eliminate the inter-observer variability of ECOG-PS; and this is in contrast to computed tomographic SMI assessment which has been shown to have a good intra-observer agreement $[21,35,36]$. ECOGPS is an important functional assessment for sarcopenia and can be combined with computed tomographic skeletal muscle analysis if required. Another limitation is the association between sarcopenia and increasing age [37]; however, the regression analysis identified SMI only to be associated with non-uptake of chemotherapy.
Our study has shown that performing SMI measurement at the time of a diagnostic CT for pancreatic cancer is feasible and can identify sarcopenia and malnourished patients who are much less likely to take up chemotherapy. This information along with ECOG-PS and age could help predict patients that are unlikely to take up palliative chemotherapy in LAPC. These patients could be triaged to initial oncology assessment for nutritional assessment prior to EUS-B referral to gain nutritional support and best supportive care and avoid unnecessary investigations.

Author Contribution MJ contributed to study design, data collection, analysis, and drafting the manuscript. JC contributed to data collection, interpretation of data, and critically revised the manuscript. JW contributed to data collection, interpretation of data, and critically revised the manuscript. AH contributed to study design, analysis, and interpretation of data and critically revised the manuscript. All authors approved the final version of the manuscript.

Availability of Data and Material The datasets used during the present study are available from the corresponding author upon reasonable request.

\section{Declarations}

Ethics Approval The study was approved by the local research ethics committee (IRAS 301193, STH 21885).

Consent to Participate The local ethical committee approved the use of anonymized historic data for the study and waived informed consent from patients.

Conflict of Interest The authors declare no competing interests.

Open Access This article is licensed under a Creative Commons Attribution 4.0 International License, which permits use, sharing, adaptation, distribution and reproduction in any medium or format, as long as you give appropriate credit to the original author(s) and the source, provide a link to the Creative Commons licence, and indicate if changes were made. The images or other third party material in this article are included in the article's Creative Commons licence, unless indicated otherwise in a credit line to the material. If material is not included in the article's Creative Commons licence and your intended use is not permitted by statutory regulation or exceeds the permitted use, you will need to obtain permission directly from the copyright holder. To view a copy of this licence, visit http://creativecommons.org/licenses/by/4.0/.

\section{References}

1. Cancer Research UK. Pancreatic cancer statistics. [cited 2020 Sep 18]. Available from: https://www.cancerresearchuk.org/health-professional/ cancer-statistics/statistics-by-cancer-type/pancreatic-cancer\# heading-Four.

2. Winter JM, et al. 1423 pancreaticoduodenectomies for pancreatic cancer: a single-institution experience. J Gastrointest Surg. 2006;10(9):1199-211.

3. Griffin OM, et al. Characterising the impact of body composition change during neoadjuvant chemotherapy for pancreatic cancer. Pancreatology. 2019. 
4. Sandini M, et al. Association between changes in body composition and neoadjuvant treatment for pancreatic cancer. JAMA Surg. 2018;153(9):809-15.

5. Santilli V, et al. Clinical definition of sarcopenia. Clin Cases Miner Bone Metab. 2014;11(3):177-80.

6. Tan BH, et al. Sarcopenia in an overweight or obese patient is an adverse prognostic factor in pancreatic cancer. Clin Cancer Res. 2009;15(22):6973-9.

7. Cooper $\mathrm{AB}$, et al. Characterization of anthropometric changes that occur during neoadjuvant therapy for potentially resectable pancreatic cancer. Ann Surg Oncol. 2015;22(7):2416-23.

8. Rollins KE, et al. The impact of sarcopenia and myosteatosis on outcomes of unresectable pancreatic cancer or distal cholangiocarcinoma. Clin Nutr. 2016;35(5):1103-9.

9. Peng P, et al. Impact of sarcopenia on outcomes following resection of pancreatic adenocarcinoma. J Gastrointest Surg. 2012;16(8):1478-86.

10. Dalal S, et al. Relationships among body mass index, longitudinal body composition alterations, and survival in patients with locally advanced pancreatic cancer receiving chemoradiation: a pilot study. J Pain Symptom Manag. 2012;44(2):181-91.

11. Sandini M, et al. Association between changes in body composition and neoadjuvant treatment for pancreatic cancer. JAMA Surg. 2018.

12. Löhr JM. Pancreatic cancer should be treated as a medical emergency. BMJ. 2014;349:g5261.

13. O'Reilly D, et al. Diagnosis and management of pancreatic cancer in adults: a summary of guidelines from the UK National Institute for Health and Care Excellence. Pancreatology. 2018;18(8):962-70.

14. Takaori K, et al. International Association of Pancreatology (IAP)/European Pancreatic Club (EPC) consensus review of guidelines for the treatment of pancreatic cancer. Pancreatology. 2016;16(1):14-27.

15. Ducreux M, et al. Cancer of the pancreas: ESMO Clinical Practice Guidelines for diagnosis, treatment and follow-up. Ann Oncol. 2015;26(Suppl 5):v56-68

16. Kumar R, et al. Multidisciplinary management of pancreatic cancer. Surg Oncol Clin N Am. 2013;22(2):265-87.

17. Kalaitzakis E, et al. Clinicians' attitudes towards endoscopic ultrasound: a survey of four European countries. Scand J Gastroenterol. 2009;44(1):100-7.

18. Cancer Research UK. Pancreatic Cancer statistics. 2021 [cited 2021 Feb 23]. Available from: https://www.cancerresearchuk.org/healthprofessional/cancer-statistics/statistics-by-cancer-type/pancreaticcancer\#heading-Five.

19. Domínguez-Muñoz JE, et al. Impact of the treatment of pancreatic exocrine insufficiency on survival of patients with unresectable pancreatic cancer: a retrospective analysis. BMC Cancer. 2018;18(1):534.

20. Védie AL, Neuzillet C. Pancreatic cancer: best supportive care. Presse Med. 2019;48(3 Pt 2):e175-85.

21. van Vugt JL, et al. A comparative study of software programmes for cross-sectional skeletal muscle and adipose tissue measurements on abdominal computed tomography scans of rectal cancer patients. J Cachexia Sarcopenia Muscle. 2017;8(2):285-97.
22. Prado CM. Body composition in chemotherapy: the promising role of CT scans. Curr Opin Clin Nutr Metab Care. 2013;16(5):525-33.

23. Martin L, et al. Cancer cachexia in the age of obesity: skeletal muscle depletion is a powerful prognostic factor, independent of body mass index. J Clin Oncol. 2013;31(12):1539-47.

24. Montano-Loza AJ, et al. Sarcopenic obesity and myosteatosis are associated with higher mortality in patients with cirrhosis. J Cachexia Sarcopenia Muscle. 2016;7(2):126-35.

25. Loh $\mathrm{KW}$, et al. Unintentional weight loss is the most important indicator of malnutrition among surgical cancer patients. Neth J Med. 2012;70(8):365-9.

26. Sikkens EC, et al. Prospective assessment of the influence of pancreatic cancer resection on exocrine pancreatic function. $\mathrm{Br}$ J Surg. 2014;101(2):109-13.

27. Kondrup J, et al. Nutritional risk screening (NRS 2002): a new method based on an analysis of controlled clinical trials. Clin Nutr. 2003;22(3):321-36.

28. Kondrup J, et al. ESPEN guidelines for nutrition screening 2002. Clin Nutr. 2003;22(4):415-21.

29. Sorensen J, et al. EuroOOPS: an international, multicentre study to implement nutritional risk screening and evaluate clinical outcome. Clin Nutr. 2008;27(3):340-9.

30. Tinju J, et al. Anthropometric, biochemical, clinical and dietary assessment for malnutrition in south Indian patients with chronic pancreatitis. Trop Gastroenterol. 2010;31(4):285-90.

31. Duggan SN, et al. The prevalence of malnutrition and fat-soluble vitamin deficiencies in chronic pancreatitis. Nutr Clin Pract. 2014;29(3):348-54.

32. Rasmussen $\mathrm{HH}$, et al. Nutrition in chronic pancreatitis. World $\mathbf{J}$ Gastroenterol. 2013;19(42):7267-75.

33. Brown KF, et al. The fraction of cancer attributable to modifiable risk factors in England, Wales, Scotland, Northern Ireland, and the United Kingdom in 2015. Br J Cancer. 2018;118(8):1130-41.

34. Jalal M, Wadsley J, Hopper A. PTU-023 Endoscopic ultrasound biopsy prior to palliative treatment for pancreatic cancer: can we prevent unnecessary procedures? Gut. 2018:A155-56.

35. Perthen JE, et al. Intra- and interobserver variability in skeletal muscle measurements using computed tomography images. Eur J Radiol. 2018;109:142-6.

36. Kelly CM, Shahrokni A. Moving beyond Karnofsky and ECOG performance status assessments with new technologies. J Oncol. 2016;2016:6186543.

37. Kim TN, Choi KM. Sarcopenia: definition, epidemiology, and pathophysiology. J Bone Metab. 2013;20(1):1-10.

Publisher's Note Springer Nature remains neutral with regard to jurisdictional claims in published maps and institutional affiliations. 